\title{
Aspectos del comportamiento de los triatominos (Hemiptera: Reduviidae), vectores de la enfermedad de Chagas
}

\author{
Diana Castillo ${ }^{1}$, Marta Wolff ${ }^{2}$ \\ ${ }^{1}$ Facultad de Ciencias Exactas y Naturales, Universidad de Antioquia, Medellín, Colombia \\ ${ }^{2}$ Departamento de Biología, Grupo Interdisciplinario de Estudios Moleculares, Universidad de Antioquia, \\ Medellin, Colombia
}

\section{Resumen}

Se presenta una revisión actualizada sobre los triatominos, vectores de la enfermedad de Chagas, en aspectos como la ubicación taxonómica de las especies actualmente reconocidas, así como de las especies encontradas en los domicilios en Colombia y del ciclo de vida del grupo en general, todo lo cual involucra el tamaño de las poblaciones, su dispersión y situaciones antrópicas que las están llevando a procesos de domiciliación. Igualmente, se hace un llamado de atención sobre las consecuencias epidemiológicas que pueden derivarse de dichas alteraciones. En Colombia, el Trypanosoma cruzi afecta el 3,3\% de la población y, aproximadamente, el 10\% se encuentra en riesgo de adquirir la infección. En esta parasitosis intervienen tres componentes biológicos: el protozoo T. cruzi, el vector triatomino Reduviidae y el reservorio vertebrado. Debido a la alteración de los ecótopos naturales a la acción devastadora de la tala de bosques, a la construcción de viviendas con hojas de palmas y a la cacería indiscriminada de animales silvestres, entre otros, la conducta de los triatominos, insectos originalmente silvestres, ha sufrido cambios con el resultado de invasiones esporádicas o permanentes a las viviendas, lo cual está originando un cambio sustancial del comportamiento de la triada vector-huésped-parásito y, consecuentemente, de la enfermedad.

Palabras clave: enfermedad de Chagas, Triatominae, comportamiento

Triatomine (Hemiptera: Rediviidae) behavioral aspects: Chagas disease vectors

\begin{abstract}
The article presents an updated review of triatomines, Chagas disease vectors, with respect to the taxonomy of presently known species, as well as those located in dwellings in Colombia, and the group's life cycle, including population size, their spreading mechanisms, and anthropic factors which are forcing domiciliation habits among them. We also call attention on the epidemiological consequences resulting from such changes. Trypanosoma cruzi in Colombia affects $3.3 \%$ of the population, from which around $10 \%$ is in risk of getting infected. Three biological components act in this infectious disease: the protozoa T. cruzi, the triatomine Reduviidae vector and the vertebrate reservoir. Although the Triatomine is originally a sylvatic insect, the alteration of its natural habitat, including forest devastation, housing construction with palm leaves and indiscriminate hunting, has changed substantially their behaviour. Thus, Triatomines are invading households and there are already signs of domiciliation which are forcing important changes in the vector-host-parasite behaviour and, consequently, in the disease itself.
\end{abstract}

Key words: Chagas disease, Triatominae, behavior

La tripanosomiasis americana o enfermedad de Chagas fue descubierta a principios del siglo por el médico brasileño Carlos Chagas, mientras trabajaba en una campaña antimalárica (1). Desde entonces, se empezó a registrar en la mayoría de los países de América y hoy está ampliamente 
distribuida, extendiéndose desde California, en el sur de los Estados Unidos, hasta el norte de Chile y Argentina. Aproximadamente 100 millones de personas están en riesgo de infectarse, 20 millones han estado o están en contacto con el parásito y 11,2 millones de personas están infectadas (2), lo que representa una prevalencia media de cerca de $4 \%$ de la población total de Latinoamérica, constituyéndose en un gran problema de salud pública en 17 países tanto en áreas rurales como urbanas (3). Según la OMS (4), en Colombia un total de 1,3 millones de personas están infectadas y alrededor de 3,6 millones están en riesgo de adquirir la infección, si se tiene en cuenta la distribución de los vectores.

\section{Ubicación taxonómica de la subfamilia Triatominae}

Los triatominos pertenecen al orden Hemíptera, el cual se caracteriza por poseer aparato bucal tipo succionador. La mayoría son organismos que se alimentan de plantas (fitófagos), algunos otros de insectos (depredadores) y unos pocos de sangre de vertebrados (hematófagos). En la suborden Heteróptera se encuentra la familia Reduviidae y la subfamilia Triatominae, única hematófaga en este grupo. De las 124 especies registradas, 111 están restringidas al Nuevo Mundo y 107 se han informado en infecciones naturales y experimentales con $T$. cruzi $(5,6)$.

La clasificación de la subfamilia Triatominae, según Lent y Wygodzinsky (7), Jurberg y Galvao (8), Lent, Jurberg y Galvao (9) es la siguiente:

Tribu: Alberproseniini Martínez y Carvallo, 1977 Género: Alberprosenia Martínez y Carvallo, 1977

Tribu: Bolboderini Usinger, 1944.

Género: Bolbodera Valdés, 1910. Belminus Stal, 1859.

Microtriatoma Prosen y Martínez, 1952. Parabelminus Lent, 1943.

Tribu: Cavernicolini Usinger, 1944.

Género: Cavernicola Barber, 1937.

Correspondencia: Apartado aéreo 1226, Medellín dcastil@matematicas.udea.edu.co

mwolff@matematicas.udea.edu.co

Recibido: 04/08/99; aceptado: 25/02/00
Tribu: Rhodiniini Pinto, 1926.

Género: Rhodnius Stal, 1859.

Psammolestes Bergroth, 1911.

Tribu: Triatomini Jeannel, 1919.

Género: Triatoma Laporte, 1832.

Panstrongylus Berg, 1879.

Eratyrus Stal, 1859.

Paratriatoma Barber, 1938.

Dipetalogaster Usinger, 1939.

Linshcosteus Distant, 1904.

Hermanlentia Jurberg y Galvao, 1997

Mepraia Mazza, Gajardo y Jord, 1940

\section{Especies de importancia epidemiológica}

Hasta 1998, se habían informado en Colombia 20 especies de triatominos (10) y en 1999 se informaron otras tres (11-13). Por su importancia epidemiológica, se destacan aquellas conocidas como domiciliadas o que invaden el peridomicilio humano: Rhodnius prolixus, Triatoma dimidiata, Triatoma maculata, Triatoma venosa (14), Rhodnius pallescens (15) y Panstrongylus geniculatus (16). $R$. prolixus es la especie de mayor importancia desde el punto de vista epidemiológico, debido a la frecuencia con que coloniza el domicilio humano y al alto porcentaje de infección por T. cruzi. Esta especie se encuentra ampliamente distribuida en los departamentos de La Guajira, Cesar, Norte de Santander, Santander, Boyacá, Arauca, Casanare, Cundinamarca, Tolima, Meta, Huila, Caquetá, Bolivar, Antioquia y Caldas (14).

\section{Hábitat}

Hoy se acepta que la tripanosomiasis americana se estableció como una zoonosis que involucra a triatominos y roedores pequeños de los valles andinos. Las civilizaciones precolombinas, al trabajar la tierra, destruyeron los ecótopos naturales, construyendo a la vez ecótopos alternativos en las viviendas humanas, que ofrecian refugio y alimento permanente. Así, algunas especies oportunistas, capaces de invadir y colonizar los domicilios, incorporaron al hombre en el ciclo de $T$. cruzi. Testimonio de este establecimiento de la antropozoonosis sería el hallazgo de antígenos de $T$. cruzi en momias del imperio incaico (17). 
Cuando el hombre penetró en los focos naturales, su acción sobre los ecótopos silvestres fue y sigue siendo devastadora por la quema y tala de bosques, la construcción de viviendas con material vegetal y la caza indiscriminada de animales silvestres, lo que ha modificado en muchas zonas del país la relación endémica natural de los componentes biológicos que intervienen en el ciclo del parásito, así como el comportamiento de los insectos triatominos, los cuales son primitivamente silvestres. Especies como R. prolixus está adaptada a las condiciones artificiales de la vivienda humana y se constituye en el principal vector de la enfermedad de Chagas en el país.

En general, los triatominos silvestres están asociados con nidos y refugios en rocas de pequeños mamíferos o aves. A menudo, la única manera de capturarlos es abordar la tarea de abrir laboriosamente los hábitats potenciales. Sin embargo, debido a que las poblaciones de triatominos selváticos suelen ser pequeñas, es posible que varias horas de trabajo sólo produzcan la captura de uno o dos ejemplares $(3,18)$.

\section{Ciclo de vida}

Los triatominos son organismos hemimetábolos cuyo ciclo biológico posee cinco estadios de ninfal, todos ellos hematófagos estrictos al igual que los adultos. Su ciclo de vida, por tanto, está condicionado por una monodieta con comidas abundantes seguidas de largos períodos de ayuno. La oviposición comienza a los 10-20 días siguientes a la cópula. Cada hembra puede depositar entre 100 y 600 huevos durante su vida adulta (3-4 meses), dependiendo de la especie y de la cantidad de alimento ingerido. Las ninfas se alimentan de los mismos hospederos que los adultos. Se puede decir que, en condiciones óptimas de laboratorio, la tasa neta de crecimiento de la población puede alcanzar 25 a 50 veces por generación; sin embargo, en la mayoría de las poblaciones domésticas es igual a 1 en promedio (19).

El estudio sobre el comportamiento de los vectores en zonas endémicas ha conducido, inicialmente, a la simulación de ambientes en el laboratorio $\mathrm{y}$, posteriormente, al estudio de la densidad en poblaciones domésticas. Este último hace referencia a los cambios en la tasa de reproducción de las hembras y al balance nutricional de los insectos como parte importante en el desarrollo de huevo a adulto (19).

\section{Comportamiento alimentario}

Según Schofield (3), la regulación natural del tamaño de la población de triatominos depende de la densidad de sus huéspedes vertebrados. A medida que aumenta el tamaño de la población y que una mayor cantidad trata de alimentarse de un número fijo de huéspedes, éstos se irritan cada vez más y hay mayor probabilidad de que los insectos interrumpan su alimentación antes de llenarse completamente. De manera típica, los triatominos tardan entre 10 y 20 minutos en alimentarse totalmente. El volumen de sangre que ingieren las ninfas puede ser de 8 a 9 veces su peso corporal; sin embargo, en adultos puede ser de 2 a 4 veces. Existe evidencia muy clara, procedente de experimentos en animales de laboratorio y voluntarios humanos, de que el huésped reacciona con irritación creciente de acuerdo con el número de pitos que se alimentan $(20,21)$. A consecuencia de la agitación del huésped, muchos insectos cesan la alimentación antes de llenarse completamente. Tal regulación denso-dependiente del tamaño de la ingestión de sangre parece representar un factor clave que influye en la regulación del tamaño de la población y la dispersión de los adultos (3). Así, a medida que aumenta la densidad, cada pito ingiere cantidades de sangre progresivamente menores. La reducción de la condición nutritiva tiene tres consecuencias principales: la velocidad del desarrollo de la ninfa disminuye, reduciendo la velocidad de paso a los estadios sucesivos, de manera que las hembras emergen más lentamente; las hembras ingieren menos cantidad de sangre, depositando por ende menos huevos, y la reducción de la ingestión de sangre por parte de los adultos machos y hembras tiende a aumentar la probabilidad de que inicien un vuelo dispersivo, lo cual permite la invasión de sitios alternativos, como las viviendas humanas.

Otra consecuencia de la oportuna disponibilidad de sangre es la deyección durante o posterior a la alimentación. Los cambios denso-dependientes del comportamiento de alimentación de los 
insectos influyen en el momento de deyección y la dinámica de transmisión de T. cruzi (19), lo cual puede afectar la probabilidad de infección, ya que los vectores más eficaces son los que defecan mientras están aún en contacto con el huésped. Sin embargo, el momento de la deyección depende tanto de la especie como de la densidad y, así mismo, de la cantidad de sangre ingerida. Los triatominos que ingieren poca sangre tienden a defecar más tarde que los que toman cantidades mayores $(22,23)$.

Como puede observarse, la fisiología y la dinámica de población de estos insectos están centradas en el proceso alimentario-reproductivo. De esta manera, el umbral, la velocidad, la duración, la intensidad y aún la dirección de los procesos fisiológicos en los triatominos, están determinados por factores externos (temperatura, presión de vapor de agua) y por factores endógenos (ingestión, reservas metabólicas, ayuno). La mayoría de las especies de triatominos se alimentan de noche, cuando sus huéspedes vertebrados están dormidos. La orientación de corta distancia hacia el huésped parece involucrar una combinación de señales, incluidas el $\mathrm{CO}_{2}$ como estímulo de alerta y el calor radiante para obtener la orientación y la horadación. Al parecer, cuando tienen hambre y están alertas, los triatominos horadan cualquier superficie cálida y prueban el fluido subyacente; si contiene «factores de nutrición» reconocibles (principalmente, nucleótidos ricos en energía, tales como el ATP), los pitos empiezan a alimentarse (24). Así pues, la temperatura y los factores independientes de la densidad, como la eliminación de los sitios de reposo, por ejemplo, los nidos en el caso de las especies selváticas, o el mejoramiento de la vivienda o la aplicación de insecticidas en el caso de las especies domésticas, tienen un efecto crucial en la ingestión $y$, por ende, en el tamaño de la población. Se ha demostrado que ni la depredación ni el parasitismo parecen tener un efecto significativo en la regulación de la densidad de la población de triatominos domésticos, pero la depredación por parte del huésped silvestre puede ser mucho más importante para la regulación de las poblaciones selváticas (3).

\section{Mecanismos de dispersión}

Los triatominos tienen dos mecanismos de dispersión, uno pasivo a través del huésped vertebrado, y otro activo por desplazamiento o por vuelo en el caso de los adultos (3). Aunque la dispersión es principalmente función del comportamiento del huésped, la dispersión activa por vuelo está asociada con el mal estado nutricional de los insectos adultos y con la regulación de la densidad de las poblaciones; por tanto, el inicio del vuelo puede considerarse como una reacción a la destrucción del hábitat y a la muerte o migración del huésped (18).

En consecuencia, cuando en un hábitat natural se eliminan las fuentes de alimento de los triatominos, tales como armadillos, marsupiales, roedores, aves, etc., y simultáneamente se invaden estos lugares en forma desordenada con la construcción de viviendas cerca del bosque y la introducción de animales domésticos, los triatominos adultos realizan inicialmente vuelos dispersivos y, finalmente, en algunos casos se adaptan a las viviendas, dado que éstas presentan condiciones ambientales estables, así como alimento disponible y permanente, ocasionando, así, un aumento descontrolado del número de triatominos que, en condiciones naturales, no se da, pues sólo sobreviven y consiguen alimento un número limitado. En las casas, en cambio, los habitantes humanos y los animales domésticos son fuentes permanentes de alimento y los insectos pueden desarrollar allí su ciclo completo, facilitando la replicación sucesiva del parásito en las zonas endémicas.

\section{Conclusiones y discusión}

Por sus hábitos hematófagos y por ser vectores del agente causal de la enfermedad de Chagas, T. cruzi, los triatominos implican un alto riesgo epidemiológico, especialmente aquellos que invaden las habitaciones humanas, ya que encuentran una situación muy favorable para su desarrollo y reproducción en ambientes cercanos al hombre. Las condiciones socioeconómicas, en especial ias de los países latinoamericanos, sumadas a las dificultades para la asistencia sanitaria, amenazan con el aumento anual en la 
población afectada por este mal, en la que se cuentan muchos niños en edad escolar.

El comportamiento dispersivo de los triatominos se ve favorecido por situaciones como la electrificación, la construcción de carreteras y por el desarrollo urbanístico en zonas rurales, lo cual desplaza no sólo los reservorios que son su fuente de alimento, sino también a los insectos mismos, que por su mal estado nutricional, vuelan en busca de otras alternativas como los animales domésticos y el hombre.

Es por esto que, actualmente, los mecanismos de control y vigilancia de la enfermedad de Chagas buscan ante todo combatir los vectores para romper el ciclo y así evitar la transmisión del parásito. En cuanto a la taxonomía, clasificación y estudios filogenéticos de los vectores, se están usando técnicas morfométricas (tradicional y geométrica) y moleculares (RAPD e isoenzimas) para conocer la estructura de la población y realizar diferenciaciones intra e interespecíficas.

Afortunadamente, existen grupos que están adelantando investigaciones sobre ecoepidemiología, genética, inmunología, biologia molecular y genética de poblaciones de la enfermedad y sus tres componentes biológicos. Además, algunos de ellos adelantan campañas educativas y de control y prevención en comunidades en riesgo de infección o ya afectadas. Es importante enfatizar el compromiso que deben tener las entidades estatales y de salud para colaborar con estas acciones de prevención y control de la enfermedad de Chagas, fortaleciendo las actividades educativas dirigidas a la participación e integración comunitaria.

\section{Referencias}

1. Briceño L. La casa enferma. Sicología de la enfermedad de Chagas. Acta Científica Venezolana 1990;149.

2. Schofield C. Control de la tripanosomiasis americana y leishmaniosis: aspectos biológicos, genéticos y moleculares. Gulh F, Jaramillo C, editores. Enfermedad de Chagas. Santa fe de Bogotá: Universidad de los Andes; 1998. p.1-4.

3. Schofield C. Triatominae: biología y control. Eurocommunica 1994. p.80.

4. Organisation Mondiale de la Santé. Lutte contre la maladie de Chagas. Serie de rapports techniques. Geneve: OMS; 1991. p.811,102.
5. Zeledón R. Effects of triatominae behavior on Trypanosoma transmission. New approaches in American trypanosomiasis research. Scientific publications. Washington, D.C.: OPS 1976. p.318.

6. Sherlock I, Carcavallo R, Galindez I. List of natural and experimental flagellate infections in several triatomine species. In: Caravallo RU, Galindez I, Jurberg J, Lent H. Rio de Janeiro: Editora Fiocruz 1998. p.393.

7. Lent $\mathbf{H}, \mathbf{W y g o d z i n s k i} \mathbf{P}$. Revision of the Triatominae (Hemiptera: Reduviidae), and their significance an vectors of Chagas disease. Bull Am Mus Nat His 1979; 163:520.

8. Jurberg J, Galvao C. Hermanlentian. Gen. Da tribo Triatomini, com um rol de especies de triatominae (Hemiptera, Reduviidae). Mem Inst Oswaldo Cruz 1997; 92:181-5.

9. Lent H, Jurberg J, Galvao C. Revalidacao do Genero Mepraia, Mazza, Gajardo \& Jorg, 1940 (Hemiptera, Reduviidae, Triatominae). Mem Inst Oswaldo Cruz 1994; 89:347-52.

10. Guhl F. Estado actual del control de la enfermedad de Chagas en Colombia. En: Guhl F, Jaramillo C, editores. Control de la tripanosomiasis americana y leishmaniosis: aspectos biológicos, genéticos y moleculares. Santa Fe de Bogotá: Universidad de Los Andes 1998. p.47-81.

11. Villegas ME, Manotas LE, Molina J, Guhl F. Primer reporte de la presencia de Rhodnius brethesi Matta, 1919 en Colombia. XXVI Congreso Sociedad Colombiana de Entomología 1999. p.68.

12. Moreno J. Nueva especie de Rhodnius, 1999 (en prensa).

13. Sandoval C, Gutiérrez R, Luna K, Joya M, Angulo V. Distribución de la enfermedad de Chagas y su infección natural con Trypanosoma cruzi y $T$. rangeli en Santander. Memorias, XXVI Congreso Sociedad Colombiana de Entomología. Santafé de Bogotá: Sociedad Colombiana de Entomologia; 1999. p.73.

14. Corredor A, Santacruz M, Páez S, Guatame L. Distribución de los triatominos domiciliarios y extradomiciliarios en Colombia. Bogotá: Instituto Nacional de Salud; 1990. p.144.

15. Moreno J. Estudios epidemiológicos sobre la enfermedad de Chagas en algunas regiones de Colombia. En: Tópicos de infectologia. Medellin: Universidad de Antioquia; 1995. p.97-104.

16. Wolff M, Arboleda J, González C, Manotas I, Rueda A. Estudio de tripanosomiasis americana, municipio de Amalfi, vereda Montebello. Bol Epid Antioquia 1994; XIX:302-5.

17. Guhl F, Jaramillo C, Yockten R, Vallejo G, CárdenasArroyo F. Trypanosoma cruzi DNA in human mummies. Lancet 1997;349(9062):1370. 
18. Miles ML. Human behaviour and the propagation of Chagas disease. Trans. R Soc Trop Med Hyg. $1976 ; 70: 521-2$

19. Schofield C, Dujardin JP, Moreno J, GuhI F. Memorias Curso Posgrado Genética poblacional de vectores triatomíneos aplicada al control vectorial de la enfermedad de Chagas. Santa Fe de Bogotá 1997;89.

20. Schofield C. A key for identifying faecal smears to detect domestic infestations of triatomine bugs. Rev Soc Brasilera Med Trop 1986;19:5-8.

21. Wier-López EH. Estado alimentario y regulación poblacional en Rhodnius prolixus (Hemiptera:
Reduviidae). Tesis, Universidad Simón Bolivar, Venezuela 1982;285.

22. Krik ML, Schofield CJ. Density-dependent timing of defaecation by Rhodnius prolixus, and its implications for the transmission of Trypanosoma cruzi. Trans $R$ Soc Trop Med Hyg 1987;81:348-9.

23. Trumper EV, Gorla DE. Density-dependent timing of defecation by Triatoma infestans. Trans R Soc Trop Med Hyg 1991:85:800-2.

24. Friend WG, Smith JJ. Factors affecting feeding by bloodsucking insects. Ann Rev Ent 1977;22:309-31. 